\title{
Struo2: efficient metagenome profiling database construction for ever-expanding microbial genome datasets
}

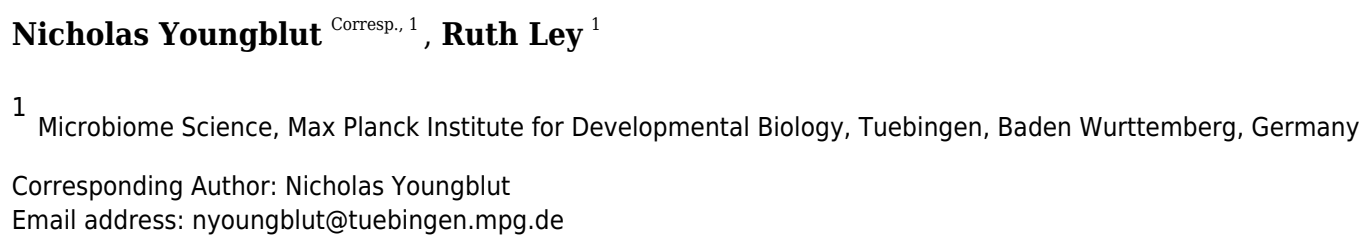

Mapping metagenome reads to reference databases is the standard approach for assessing microbial taxonomic and functional diversity from metagenomic data. However, public reference databases often lack recently generated genomic data such as metagenome-assembled genomes (MAGs), which can limit the sensitivity of read-mapping approaches. We previously developed the Struo pipeline in order to provide a straightforward method for constructing custom databases; however, the pipeline does not scale well enough to cope with the ever-increasing number of publicly available microbial genomes. Moreover, the pipeline does not allow for efficient database updating as new data are generated. To address these issues, we developed Struo2, which is $>3.5$ fold faster than Struo at database generation and can also efficiently update existing databases. We also provide custom Kraken2, Bracken, and HUMAnN3 databases that can be easily updated with new genomes and/or individual gene sequences. Efficient database updating, coupled with our pre-generated databases, enables "assembly-enhanced" profiling, which increases database comprehensiveness via inclusion of native genomic content. Inclusion of newly generated genomic content can greatly increase database comprehensiveness, especially for understudied biomes, which will enable more accurate assessments of microbiome diversity. 


\section{Struo2: efficient metagenome profiling database \\ 2 construction for ever-expanding microbial genome datasets}

3

4

5

6

7 8

9

10

11

12

13

14

15

16

17

18
Nicholas D. Youngblut ${ }^{1}$, Ruth E. Ley ${ }^{1}$

${ }^{1}$ Department of Microbiome Science, Max Planck Institute for Developmental Biology, Tübingen, Baden Württemberg, Germany

Corresponding author:

Nicholas Youngblut ${ }^{1}$

Department of Microbiome Science, Max Planck Institute for Developmental Biology, Max

Planck Ring 5, 72076 Tübingen, Baden Württemberg, Germany

Email address: nicholas.youngblut@tuebingen.mpg.de 
19

20

21

22

23

24

25

26

27

28

29

30

31

32

33

34

35

36

37

38

39

40

41

42

43

44

45

46

47

48

49

50

51

52

53

54

55

56

\section{Abstract}

Mapping metagenome reads to reference databases is the standard approach for assessing microbial taxonomic and functional diversity from metagenomic data. However, public reference databases often lack recently generated genomic data such as metagenome-assembled genomes (MAGs), which can limit the sensitivity of read-mapping approaches. We previously developed the Struo pipeline in order to provide a straight-forward method for constructing custom databases; however, the pipeline does not scale well enough to cope with the ever-increasing number of publicly available microbial genomes. Moreover, the pipeline does not allow for efficient database updating as new data are generated. To address these issues, we developed Struo2, which is $>3.5$ fold faster than Struo at database generation and can also efficiently update existing databases. We also provide custom Kraken2, Bracken, and HUMAnN3 databases that can be easily updated with new genomes and/or individual gene sequences. Efficient database updating, coupled with our pre-generated databases, enables "assembly-enhanced" profiling, which increases database comprehensiveness via inclusion of native genomic content. Inclusion of newly generated genomic content can greatly increase database comprehensiveness, especially for understudied biomes, which will enable more accurate assessments of microbiome diversity. Availability:

- Struo2: https://github.com/leylabmpi/Struo2

- Pre-built databases: http://ftp.tue.mpg.de/ebio/projects/struo2/

- Utility tools: https://github.com/nick-youngblut/gtdb to taxdump

\section{Introduction}

Utilizing shotgun metagenomics for microbiome research is increasing in popularity due to the reduced costs and scalability of sequencing and the improved ease of sequence library generation (Sczyrba et al. 2017; Breitwieser, Lu, and Salzberg 2019). Metagenome profiling is a very common approach of assessing microbial diversity from metagenomics data, which involves mapping unassembled reads or assembled contigs to reference databases in order to derive annotations for taxonomy, metagenomic pathways, genes, and other genomic content. Importantly, metagenome profiling requires the use of $\geq 1$ reference database, which are all incomprehensive and are often biased toward pathogens and other well-studied microbes (Breitwieser, Lu, and Salzberg 2019; Loeffler et al. 2020). While microbial diversity can be directly assessed via kmer-based approaches that do not rely on reference databases (Benoit et al. 2016; Rowe et al. 2019), such methods provide no direct insight into the taxa or genomic content that differs across treatment groups or environmental gradients.

Most metagenome profiling software includes "standard" reference databases (Kim et al. 2016; Franzosa et al. 2018; Wood, Lu, and Langmead 2019; LaPierre et al. 2020). For instance, the popular HUMANnN pipeline includes multiple databases for assessing both taxonomy and function from read data (Franzosa et al. 2018). Similarly, Kraken2 includes a set of standard databases for taxonomic classification of specific clades (e.g., fungi or plants) or all taxa (Wood, 
$57 \mathrm{Lu}$, and Langmead 2019). While such standard reference databases provide a crucial resource for

58

59

60

61

62

63

64

65

66

67

68

69

70

71

72

73

74

75

76

77

78

79

80

81

82

83

84

85

86

87

88

89

90

91

92

93

94

95 always be somewhat out-of-date, given the rapid increase in newly assembled genomic content. For instance, database biases and lack of representation can be reduced by first assembling genes and genomes from metagenomes and adding the new genomic content to the existing databases (Youngblut et al. 2020). Second, standard profiling databases may not be customized for specific research questions such as smaller databases focused on certain (well-curated) taxa or genomic content from specific environments (e.g., only marine or soil microbiomes). Third, the underlying taxonomy or genomic annotations included in the database may be suboptimal. For example, the Genome Taxonomy Database (GTDB) defines a taxonomy based on microbial genome phylogenies, which is highly standardized relative to the polyphasic taxonomy of the NCBI; however, the GTDB taxonomy is not available in standard profiling databases (Parks et al. 2020; de la Cuesta-Zuluaga, Ley, and Youngblut 2020).

The process of making custom reference databases is often complicated and requires substantial computational resources, which led us to create Struo for straight-forward custom metagenome profiling database generation (de la Cuesta-Zuluaga, Ley, and Youngblut 2020). We utilized our toolset to generate and publish custom metagenome profiling databases from the GTDB, which not only provides a standardized taxonomy but also increased the percent of gut metagenome reads mapped to database references relative to standard Kraken2 and HUMAnN2 databases.

While Struo greatly aids in custom database generation, it does require substantial computational resources, especially for the gene annotation that is necessary for creating custom HUMAnN databases. Specifically, Struo requires $\sim 2.4$ CPU hours per genome and thus took $>51,000$ CPU hours when applied to $\sim 21,000$ species-representative genomes in Release 89 of the GTDB. The number of representative genomes has increased to $>31,900$ in the subsequent GTDB release (an $\sim 50 \%$ increase), which would require $>77,900$ CPU hours ( $>9.1$ years) for the Struo pipeline. Given that the number of genomes added to the GTDB and other databases is rapidly expanding (Fig. 1) (Almeida et al. 2020; Sayers et al. 2021), we sought to develop a pipeline that scales accordingly. Struo2 is a rewrite and expansion of Struo, which is substantially faster, can efficiently update existing databases, and includes generally applicable utility tools for database manipulation.

\section{Materials \& Methods}

Struo2 database creation algorithm

A general outline of the Struo2 algorithm is shown in Figure 2A. Struo2 can generate database files for 4 main database types: "Kraken2", "Bracken", "genes", and "HUMAnN3" (Wood, Lu, and Langmead 2019; Lu et al. 2017; Franzosa et al. 2018). Struo2 uses snakemake and conda (Köster and Rahmann 2012), and so there are no dependencies that must be installed prior to pipeline execution besides snakemake, conda, and pandas (for input table loading). Moreover, snakemake allows for efficient job execution and easy scaling on to high performance

Peerj reviewing PDF | (2021:07:64023:0:1:NEW 29 Jul 2021) 
96

97

computing systems. We note that the Struo2 pipeline code is a substantial re-write and expansion of the original Struo pipeline (e.g., $\sim 1500$ versus $\sim 7000$ lines of code in Struo versus Struo2, respectively). Struo2 has extensive documentation, including tutorials on its usage.

The user input for Struo2 database creation is a table that lists: i) unique taxon names, ii) assembly accession identifiers (if available), iii) paths to (compressed) genome assembly fasta files, iv) taxonomy identifiers (taxids) used for Kraken2 database construction, and v) taxonomies at the genus and species levels (used for HUMAnN3). We provide 2 utility scripts to aid in construction of custom databases from genomes in the GTDB: GTDB_metadata_filter.R and genome_download.R. GTDB_metadata_filter.R can filter the publicly available GTDB archaeal and bacterial genome metadata files to a select subset of genomes (e.g., those with a lower CheckM-estimated contamination). genome_download.R can then download all of the user-selected GTDB genomes and add the path to the genome assembly fasta files to the GTDB metadata table. This updated metadata table can then be directly used as input to GTDB.

For construction of the custom Kraken2 database, contigs are renamed to "kraken:taxid $|<\operatorname{taxid}>|<$ seqid $>$ ", as described in the Kraken2 manual (https://github.com/DerrickWood/kraken2/wiki/Manual). The renamed contigs are added to a new Kraken2 database via kraken-build, and then the database is constructed via the same command. By default, the GTDB taxonomy is used, which entails providing custom GTDB taxdump files created via the gtdb_to_taxdump.py utility tool (available at https://github.com/nick-youngblut/gtdb to taxdump). The "taxonomy" and "library" directories created by Kraken 2 for temporary file storage are saved in order to expedite database updating with new genomes.

Custom Bracken database files are created for any number of read lengths that the user specifies (100 and 150 base pairs by default). The bracken-build.py script is used within the pipeline for constructing each Bracken database.

In order to construct a custom HUMAnN3 database, Struo2 first creates a precursor "genes" database, which consists of gene sequences from each genome and gene clusters generated via mmseqs linclust. To construct the "genes" database, genes are first called via prodigal (Hyatt et al. 2010), and then de-replicated at 97\% sequence identity with vsearch (Rognes et al. 2016), which is similar to the standard HUMAnN database construction process (Franzosa et al. 2018). Non-redundant gene sequences from all genomes are combined, and the metadata of each gene sequence (e.g., genome of origin, contig of origin, and location on the contig) is also combined into one text file. The amino acid gene sequences are clustered via mmseqs linclust. By default, gene cluster representative sequences are annotated against UniRef90 (version 2019-01; the same as used by HUMAnN3) via mmseqs search with 2 search iterations and 3 sensitivity steps $(\min =1, \max =6)$. Prior to annotation, the sequence queries are split into $n$ batches and run in parallel for faster distributed searching with snakemake ( $n$ is userdefined). For each gene cluster, the UniRef90 annotations are propagated to each gene. UniRef90 annotations are mapped to UniRef50 identifiers via a mapping file created from the UniRef90.xml file available from the UniProt ftp server 
136 (ftp://ftp.uniprot.org/pub/databases/uniprot/). The unirefxml2clust50-90idx.py utility script is

137

138

139

140

141

142

143

144

145

146

147

148

149

150

151

152

153

154

155

156

157

158

159

160

161

162

163

164

165

166

167

168

169

170

171

172

173

174

used to generate this mapping file (available at https://github.com/nickyoungblut/gtdb to taxdump). The mapping of UniRef90 to UniRef50 identifiers obviates the need to annotate genes separately against UniRef90 and UniRef50. We note that Struo requires separate rounds of annotation to each UniRef database instead of this UniRef90-to-UniRef50 mapping approach, which greatly increases the run time versus Struo2 when the goal is to obtain annotations for both UniRef 90 and UniRef50. Note that the genes database includes both nucleotide and amino acid sequences for each gene.

The annotated gene sequences are renamed in the format “ $<$ UniRefID $>\mid<$ gene_length $>\mid g \_<$genus $>$; _ _ $<$species $>$” for creation of the HUMAnN3 database. Note that the taxonomy information is provided by the user in the original input table. bowtie2-build and diamond makedb are used to generate a HUMAnN3-compatible bowtie2 and DIAMOND databases of all annotated gene nucleotide and amino acid sequences, respectively.

\section{Struo2 database update algorithm}

Struo2 can update existing Struo2-generated Kraken2, Bracken, genes, and HUMAnN3 databases. The databases can be updated with new genomes or individual gene sequences (e.g., created via metagenome assembly with PLASS (Steinegger, Mirdita, and Söding 2019)).

If the input is a set of new genomes, the input is essentially the same as for database creation, except the existing database files must also be provided. Database updating with individual gene sequences requires the gene sequences in amino acid format (and also nucleotide, if available) and metadata on each gene (i.e., the genus- and species-level taxonomy inferred via mmseqs taxonomy or other approaches).

Kraken 2 custom databases are updated via adding more genomes to the existing library via kraken-build. New Bracken databases are created from the updated Kraken2 database.

Gene sequences, either originating from new genomes or new individual sequences, are added to the existing mmseqs gene cluster database via mmseqs clusterupdate. Newly formed clusters are annotated with mmseqs search, while existing annotations are used for existing clusters. The updated database of annotated genes are used for creating new HUMAnN3compatible bowtie2 and DIAMOND databases.

We note that database updating does not require consistent genomic representation from each representative genome (e.g., the same taxonomic marker genes), given that neither Kraken2/Bracken nor HUMAnN3 require such consistency.

\section{Benchmarking custom database construction and updating}

We used genomes from the GTDB (Release 95) for all benchmarking. Only genomes with $\geq 50 \%$ CheckM-estimated completeness, $<5 \%$ CheckM-estimated contamination were included (Parks et al. 2015). To reduce biases towards species with large numbers of representative genomes, we selected one genome per species. The genome with the 
175 highest estimated completeness and lowest estimated contamination was selected for all

176

177

178

179

180

181

182

183

184

185

186

187

188

189

190

191

192

193

194

195

196

197

198

199

200

201

202

203

204

205

206

207

208

209

210

211

212

213

candidates of each species. The final pool consisted of 30,989 genomes (Fig. S2).

We used the same genome subsets for benchmarking database creation with both Struo and Struo2. We benchmarked the combined time to generate Kraken2, Bracken, and HUMAnN databases, which included both UniRef50 and UniRef90 annotations for the HUMAnN databases. Struo was run with default parameters. Both pipelines were run on the same computational architecture, consisting of a high performance computing cluster comprising nodes running Ubuntu 18.04.5 with AMD Epyc CPUs and 0.5-2 terabytes of RAM. The CPU hours shown in Figure 2B are the sum of all CPU hours for all snakemake jobs, as recorded via snakemake's benchmarking feature.

We only benchmarked database updating for Struo2, given that Struo cannot update databases, and we also clearly show in Figure 2B that database generation is much slower for Struo. We first used Struo2 to generate custom Kraken2, Bracken, and HUMAnN databases from 1000 genomes. These "n1000" databases were used for all database update benchmarking. The genomes used for database update benchmarking did not overlap with any genomes used to generate the n1000 databases, and they did not overlap with each other. We used subsets of 10 , $100,175,250,350$, and 500 genomes. We used the linear regression models shown in Figure 2B to estimate the CPU hours that would be required to generate each database from scratch rather than updating.

Benchmarking custom database accuracy as a function of genome assembly quality We utilized two randomly chosen sets of 100 genomes from the GTDB r95 reference genome pool (described above). For each set, we simulated varying levels of misassemblies among each genome in the set, with the same number of misassemblies introduced into each genome per simulation set. Simulations were created via a custom python script: genome_misasmbl_sim.py (available in the Struo2 GitHub repository). Three types of misassemblies were simulated: breakpoints (splitting a contig into 2 pieces), rearrangements (relocating a genomic fragment within one contig to another location on the same contig or other contig in the assembly), and chimerisms (relocating a genomic fragment from one contig in a donor genome to a contig in a recipient genome). Breakpoint locations were selected from a uniform distribution. Fragment sizes of rearrangements and chimerisms were selected from a uniform distribution, with size a range of 1e3-1e4. We used CheckM to assess assembly quality of each genome in each synthetic genome dataset (Parks et al. 2015). Each resultant synthetic genome dataset was used to construct reference metagenome profiling databases via Struo2.

We utilized the Critical Assessment of Metagenome Interpretation (CAMI) "HMP Gut" metagenome dataset to assess how genome assembly quality affects Kraken2/Bracken taxonomic assignments (Sczyrba et al. 2017). The dataset was downloaded from the CAMI challenge website: https://data.cami-challenge.org/. The Illumina paired-end reads were subsampled to 1 million per sample. Beta diversity was calculated from the Bracken output via QIIME2

Peer) reviewing PDF | (2021:07:64023:0:1:NEW 29 Jul 2021) 
214 (Bokulich et al. 2018). Beta diversity distance matrices were compared via Mantel tests with 999

215 permutations and the Pearson correlation method.

216

217 Struo2 databases from GTDB releases 95 and 202

218 The genomes selected were as reported for the benchmarking of Struo and Struo2. The

219 custom Kraken2, Bracken, genes, and HUMAnN3 databases, are available at:

220 http://ftp.tue.mpg.de/ebio/projects/struo2/. We will publish new versions of each database as new

221 releases of the GTDB are published.

222

223

\section{Utility tools}

224

225

We have generated a set of utility tools for aiding in the construction of input for Struo2

226

227

228

229

230

231

and generally facilitating the integration of the GTDB taxonomy into existing bioinformatics

pipelines. Some of these tools are described elsewhere in the Supplement Methods. We note 2 utility tools that can have a broad applicability: gtdb_to_taxdump.py and ncbi-gtdb_map.py. The former can convert the GTDB taxonomy, as documented in the GTDB bacterial and archaeal metadata table, to NCBI-formatted taxdump files. These taxdump files can be used with any existing software that requires taxdump files, such as taxonkit (Shen and Xiong 2019) or KrakenUniq (Breitwieser, Baker, and Salzberg 2018). ncbi-gtdb_map.py maps between NCBI

232 and GTDB taxonomies, based on the taxonomy information provided in the GTDB archaeal and

233 bacterial metadata files. This tool can be useful for converting GTDB-Tk classifications to NCBI

234 taxonomies (Chaumeil et al. 2019), or converting existing NCBI taxonomies to GTDB

235

236

237

238

239 taxonomies without requiring re-classification.

\section{Code availability}

The Struo2 pipeline code and all Jupyter Notebooks describing the analyses in this study are available on GitHub at https://github.com/leylabmpi/Struo2.

240

241

242

243

244

245

246

247

248

249

250

251

252

\section{Results and Discussion}

Struo2 generates Kraken2 and Bracken databases similarly to Struo (Lu et al. 2017; Wood, Lu, and Langmead 2019), but the algorithms diverge substantially for the time consuming step of gene annotation required for HUMAnN database construction. Struo2 performs gene annotation by clustering all gene sequences of all genomes using the rapid mmseqs 2 linclust algorithm, and then each gene cluster representative is annotated via mmseq2 search (Fig. 2A; Supplemental Methods) (Steinegger and Söding 2017, 2018). In contrast, Struo annotates all non-redundant genes of each genome with DIAMOND (Buchfink, Xie, and Huson 2015). Struo2 utilizes snakemake and conda, which allows for easy installation of all dependencies and simplified scaling to high performance computing systems (Köster and Rahmann 2012).

Benchmarking on genome subsets from the GTDB showed that Struo2 requires $\sim 0.67$ CPU hours per genome versus $\sim 2.4$ for Struo (Fig. 2B), which is a $>3.5$ fold decrease. Notably, Struo 2 annotates slightly more genes than Struo, possibly due to the sensitivity of the mmseqs 
253

254

255

256

257

258

259

260

261

262

263

264

265

266

267

268

269

270

271

272

273

274

275

276

277

278

279

280

281

282

283

284

285

286

287

288

289

290

291

search iterative search algorithm (Fig. 2C). The use of mmseqs2 allows for efficient database updating of new genomes and/or individual gene sequences via mmseqs clusterupdate (Fig. S1); we show that this approach saves $15-19 \%$ of the CPU hours relative to generating a database from scratch, with a linear trend towards increased efficiency as the number of genomes are added (Fig. 2D).

We assessed how Struo2-generated databases can be affected by the assembly quality of the reference genomes used. For this assessment, we generated synthetic reference genome datasets, in which 3 types of misassemblies were introduced into each genome: breakpoints, relocations, and chimerisms (see Methods). As we increased the number of per-genome misassemblies, CheckM-estimated completeness and contamination values substantially declined and increased, respectively (Fig. 3A). The percent of annotated genes, relative to the ground truth reference genomes, declined linearly and steeply as median assembly completeness declined (linear regression, $n=10, \mathrm{R}^{2}=0.97$ ) and contamination increased (linear regression, $n=10, \mathrm{R}^{2}=$ 0.9 ; Fig. 3B). Even at a median completeness of $95 \%$ and a median contamination of $1.5 \%$, the percent of genes annotated was reduced by $25 \%$ relative to the ground truth, indicating that assembly quality has a substantial impact on gene database quality. The percent of correctly annotated genes (i.e., the correct UniRef90 ID versus the ground truth) was also linearly associated with assembly quality (linear regression, $n=10$, completeness: $\mathrm{R}^{2}=0.87$, contamination: $\mathrm{R}^{2}=0.7$ ), but the decline in annotation quality was more severe: only $\sim 60 \%$ of genes were correctly annotated when median completeness dropped to $\sim 92 \%$ (Fig. 3C). In contrast, Kraken2/Bracken database quality was not substantially affected by assembly quality, in regards to beta diversity relative to the ground truth (Fig. 3D). Beta diversity divergence increased only slightly as misassemblies increased (Mantel, permutations $=999, \rho>0.98$ for all tests). The robustness of the Kraken2/Bracken databases is likely due to the use of kmers derived from entire genomes instead of using annotated genes, as used by HUMAnN3. In summary, we recommend using high quality assemblies for custom database construction, especially for the creation of the HUMAnN3 database.

We used Struo2 to create publicly available Kraken2, Bracken, and HUMAnN3 custom databases from releases 95 and 202 of the GTDB (see Supplemental Methods). We note that the reference genomes selected from releases 95 and 202 had a median CheckM-estimated completeness of 98.5 and $97.3 \%$, respectively. The median estimated contamination was 0.71 and $0.79 \%$, respectively. Thus, our custom GTDB databases should be of high quality. We will continue to publish these custom databases as new GTDB versions are released. The databases are available at http://ftp.tue.mpg.de/ebio/projects/struo2/. We also created a set of utility tools for i) generating NCBI-formatted taxdump files from the GTDB taxonomy, ii) mapping between the NCBI and GTDB taxonomies, and iii) generating DIAMOND databases for any set of GTDB reference genomes. The taxdump files are utilized by Struo2, but these tools can be used more generally to integrate the GTDB taxonomy into existing pipelines designed for the NCBI taxonomy (available at https://github.com/nick-youngblut/gtdb to taxdump).

Peer) reviewing PDF | (2021:07:64023:0:1:NEW 29 Jul 2021) 
292

293

294

295

296

297

298

299

300

301

302

303

304

305

306

307

308

309

310

311

312

313

314

315

316

317

318

319

320

321

322

323

324

325

326

327

328

329

In summary, Struo2 provides a substantial improvement over the state-of-the-art, which is needed in order to scale custom database generation with the ever-increasing amount of available genomic data. The efficient database updating feature of Struo2 enables the following "assembly enhanced" taxonomic profiling workflow. First, the user assembles MAGs and/or genes from the metagenomes novel to the user's own study. Second, the user updates our pregenerated GTDB-based profiling databases with the newly generated genomic content. Third, the user taxonomically profiles the metagenome reads from the user's study against the newly customized databases in order to generate microbial diversity assessments that are less biased and more representative of the microbiome diversity in the microbiomes included in the study. This "assembly-enhanced" profiling method could greatly improve database representation for less-studied environments such as the gut microbiome of non-mammalian vertebrates or underrepresented human populations (Porras and Brito 2019; Youngblut et al. 2020).

\section{Acknowledgements}

We thank Albane Ruaud, Liam Fitzstevens, Jacobo de la Cuesta-Zuluaga, and Jillian Waters for providing helpful comments on an earlier version of this manuscript. This study was supported by the Max Planck Society.

\section{Data availability}

Struo2 is available at https://github.com/leylabmpi/Struo2, the pre-built databases can be found at http://ftp.tue.mpg.de/ebio/projects/struo2/, code associated with this study is available at and https://github.com/leylabmpi/Struo2, utility tools are located at https://github.com/nickyoungblut/gtdb to taxdump and https://github.com/leylabmpi/Struo2.

\section{References}

Almeida, Alexandre, Stephen Nayfach, Miguel Boland, Francesco Strozzi, Martin Beracochea, Zhou Jason Shi, Katherine S. Pollard, et al. 2020. "A Unified Catalog of 204,938 Reference Genomes from the Human Gut Microbiome.” Nature Biotechnology, July. https://doi.org/10.1038/s41587-020-0603-3.

Benoit, Gaëtan, Pierre Peterlongo, Mahendra Mariadassou, Erwan Drezen, Sophie Schbath, Dominique Lavenier, and Claire Lemaitre. 2016. "Multiple Comparative Metagenomics Using Multiset K-Mer Counting." PeerJ Computer Science 2 (November): e94.

Bokulich, Nicholas A., Benjamin D. Kaehler, Jai Ram Rideout, Matthew Dillon, Evan Bolyen, Rob Knight, Gavin A. Huttley, and J. Gregory Caporaso. 2018. "Optimizing Taxonomic Classification of Marker-Gene Amplicon Sequences with QIIME 2's q2-Feature-Classifier Plugin." Microbiome 6 (1): 90.

Breitwieser, F. P., D. N. Baker, and S. L. Salzberg. 2018. "KrakenUniq: Confident and Fast Metagenomics Classification Using Unique K-Mer Counts.” Genome Biology 19 (1): 198. Breitwieser, F. P., J. Lu, and S. L. Salzberg. 2019. "A Review of Methods and Databases for Metagenomic Classification and Assembly." Briefings in Bioinformatics. 
330

331

332

333

334

335

336

337

338

339

340

341

342

343

344

345

346

347

348

349

350

351

352

353

354

355

356

357

358

359

360

361

362

363

364

365

366

367

368

369

370

371

372

373

374

375

https://academic.oup.com/bib/article-abstract/20/4/1125/4210288.

Buchfink, Benjamin, Chao Xie, and Daniel H. Huson. 2015. "Fast and Sensitive Protein Alignment Using DIAMOND.” Nature Methods 12 (1): 59-60.

Chaumeil, Pierre-Alain, Aaron J. Mussig, Philip Hugenholtz, and Donovan H. Parks. 2019. "GTDB-Tk: A Toolkit to Classify Genomes with the Genome Taxonomy Database."

Bioinformatics , November. https://doi.org/10.1093/bioinformatics/btz848.

Cuesta-Zuluaga, Jacobo de la, Ruth E. Ley, and Nicholas D. Youngblut. 2020. "Struo: A Pipeline for Building Custom Databases for Common Metagenome Profilers." Bioinformatics 36 (7): 2314-15.

Franzosa, Eric A., Lauren J. McIver, Gholamali Rahnavard, Luke R. Thompson, Melanie Schirmer, George Weingart, Karen Schwarzberg Lipson, et al. 2018. "Species-Level Functional Profiling of Metagenomes and Metatranscriptomes." Nature Methods 15 (11): 962-68.

Hyatt, Doug, Gwo-Liang Chen, Philip F. Locascio, Miriam L. Land, Frank W. Larimer, and Loren J. Hauser. 2010. "Prodigal: Prokaryotic Gene Recognition and Translation Initiation Site Identification.” BMC Bioinformatics 11 (March): 119.

Kim, Daehwan, Li Song, Florian P. Breitwieser, and Steven L. Salzberg. 2016. "Centrifuge: Rapid and Sensitive Classification of Metagenomic Sequences.” Genome Research, October. https://doi.org/10.1101/gr.210641.116.

Köster, Johannes, and Sven Rahmann. 2012. "Snakemake--a Scalable Bioinformatics Workflow Engine." Bioinformatics 28 (19): 2520-22.

LaPierre, Nathan, Mohammed Alser, Eleazar Eskin, David Koslicki, and Serghei Mangul. 2020. "Metalign: Efficient Alignment-Based Metagenomic Profiling via Containment Min Hash." Genome Biology 21 (1): 242.

Loeffler, Caitlin, Aaron Karlsberg, Lana S. Martin, Eleazar Eskin, David Koslicki, and Serghei Mangul. 2020. "Improving the Usability and Comprehensiveness of Microbial Databases." BMC Biology 18 (1): 37.

Lu, Jennifer, Florian P. Breitwieser, Peter Thielen, and Steven L. Salzberg. 2017. "Bracken: Estimating Species Abundance in Metagenomics Data." PeerJ Computer Science 3 (January): e104.

Parks, Donovan H., Maria Chuvochina, Pierre-Alain Chaumeil, Christian Rinke, Aaron J. Mussig, and Philip Hugenholtz. 2020. "A Complete Domain-to-Species Taxonomy for Bacteria and Archaea." Nature Biotechnology 38 (9): 1079-86.

Parks, Donovan H., Michael Imelfort, Connor T. Skennerton, Philip Hugenholtz, and Gene W. Tyson. 2015. "CheckM: Assessing the Quality of Microbial Genomes Recovered from Isolates, Single Cells, and Metagenomes." Genome Research 25 (7): 1043-55.

Porras, Ana Maria, and Ilana Lauren Brito. 2019. "The Internationalization of Human Microbiome Research." Current Opinion in Microbiology 50 (August): 50-55.

Rognes, Torbjørn, Tomáš Flouri, Ben Nichols, Christopher Quince, and Frédéric Mahé. 2016. "VSEARCH: A Versatile Open Source Tool for Metagenomics." PeerJ 4 (October): e2584.

Rowe, Will Pm, Anna Paola Carrieri, Cristina Alcon-Giner, Shabhonam Caim, Alex Shaw, Kathleen Sim, J. Simon Kroll, Lindsay J. Hall, Edward O. Pyzer-Knapp, and Martyn D. Winn. 2019. "Streaming Histogram Sketching for Rapid Microbiome Analytics." Microbiome 7 (1): 40.

Sayers, Eric W., Jeffrey Beck, Evan E. Bolton, Devon Bourexis, James R. Brister, Kathi Canese, Donald C. Comeau, et al. 2021. "Database Resources of the National Center for 
376

377

378

379

380

381

382

383

384

385

386

387

388

389

390

391

392

393

394

395

396

397

Biotechnology Information." Nucleic Acids Research 49 (D1): D10-17.

Sczyrba, Alexander, Peter Hofmann, Peter Belmann, David Koslicki, Stefan Janssen, Johannes Dröge, Ivan Gregor, et al. 2017. "Critical Assessment of Metagenome Interpretation-a Benchmark of Metagenomics Software." Nature Methods 14 (11): 1063-71.

Shen, Wei, and Jie Xiong. 2019. "TaxonKit: A Cross-Platform and Efficient NCBI Taxonomy Toolkit.” Cold Spring Harbor Laboratory. https://doi.org/10.1101/513523.

Steinegger, Martin, Milot Mirdita, and Johannes Söding. 2019. "Protein-Level Assembly Increases Protein Sequence Recovery from Metagenomic Samples Manyfold." Nature Methods 16 (7): 603-6.

Steinegger, Martin, and Johannes Söding. 2017. "MMseqs2 Enables Sensitive Protein Sequence Searching for the Analysis of Massive Data Sets." Nature Biotechnology 35 (11): 1026-28. 2018. "Clustering Huge Protein Sequence Sets in Linear Time." Nature Communications 9 (1): 2542.

Wood, Derrick E., Jennifer Lu, and Ben Langmead. 2019. "Improved Metagenomic Analysis with Kraken 2." Genome Biology 20 (1): 257.

Youngblut, Nicholas D., Jacobo de la Cuesta-Zuluaga, Georg H. Reischer, Silke Dauser, Nathalie Schuster, Chris Walzer, Gabrielle Stalder, Andreas H. Farnleitner, and Ruth E. Ley. 2020. "Large-Scale Metagenome Assembly Reveals Novel Animal-Associated Microbial Genomes, Biosynthetic Gene Clusters, and Other Genetic Diversity." mSystems 5 (6). https://doi.org/10.1128/mSystems.01045-20. 
Figure 1

The number of microbial genomes and taxonomic lineages in the Genome Taxonomy Database (GTDB) is rapidly expanding.

A) The number of total genomes and GTDB taxonomic lineages in each GTDB release. B) The number of unique groups per taxonomic level in each GTDB release. Data was obtained from the GTDB ftp site: https://data.gtdb.ecogenomic.org/. 


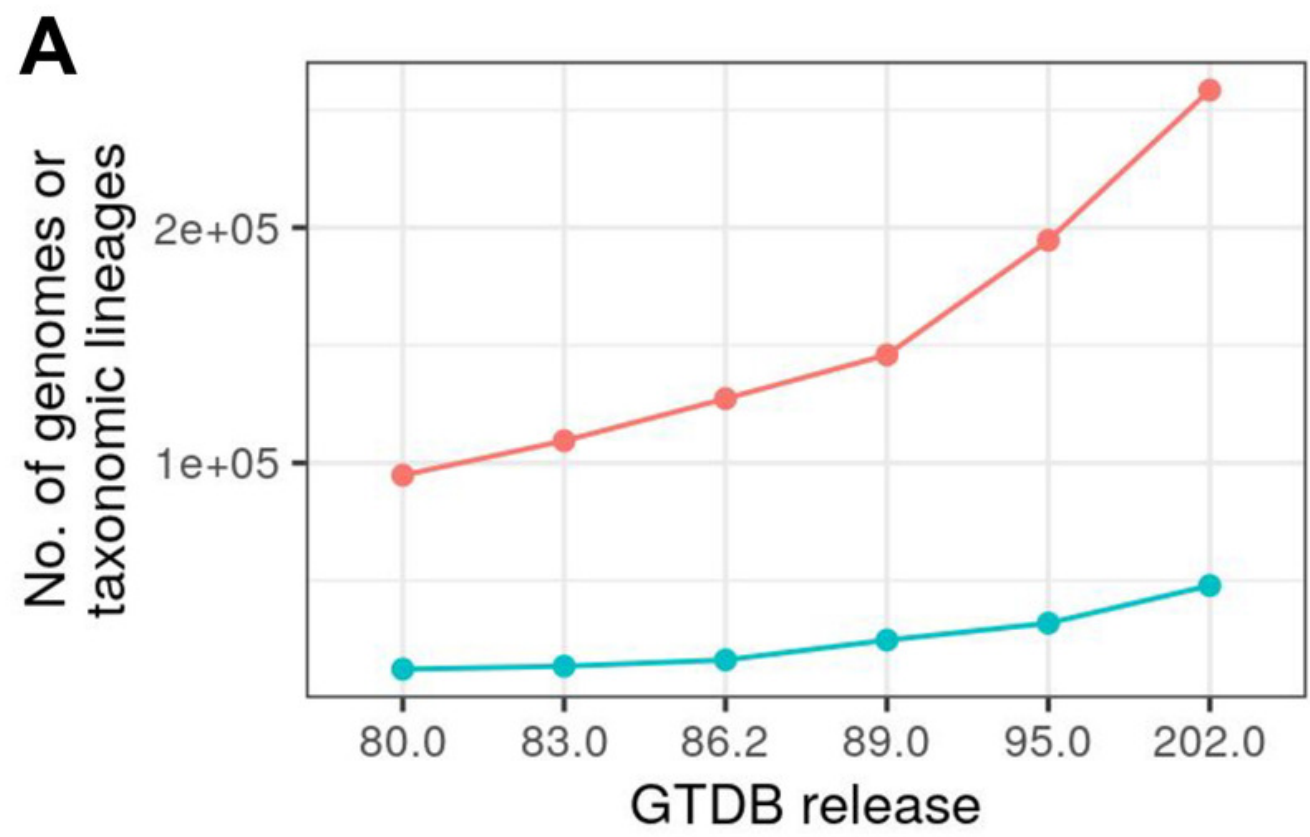

category

$\rightarrow$ genomes

$\rightarrow$ lineages

B

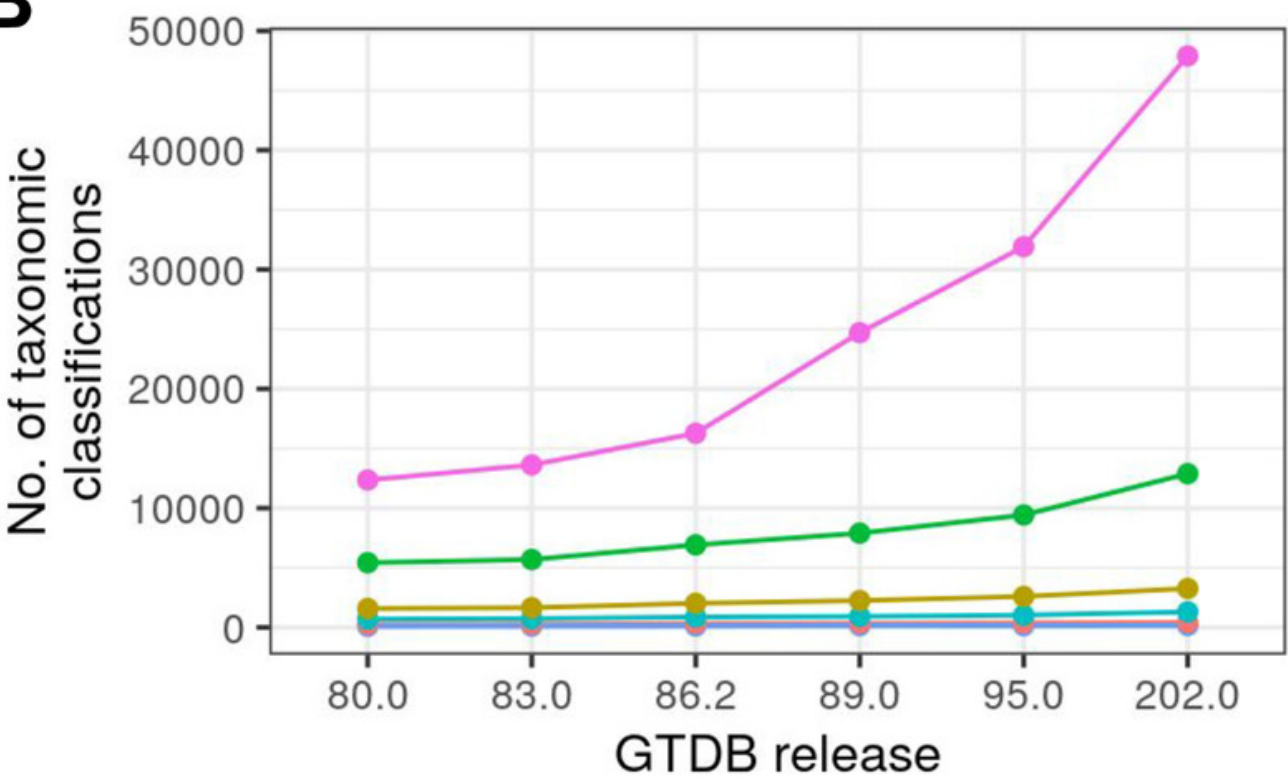

category

- Classes

- Families

$\bullet$ - Genera

$\rightarrow$ Orders

$\rightarrow$ Phyla

$\rightarrow$ Species

GTDB release 


\section{Figure 2}

Struo2 can build databases faster than Struo and can efficiently update the databases.

A) A general outline of the Struo2 database creation algorithm. Cylinders are input or output files, squares are processes, and right-tilted rhomboids are intermediate files. The largest change from Struo is the utilization of mmseqs 2 for clustering and annotation of genes. B) Benchmarking the amount of CPU hours required for Struo and Struo2, depending on the number of input genomes. The equations show that Struo2 scales $\sim 3.5$ fold faster than Struo1. C) The number of genes annotated with a UniRef90 identifier, which shows that both Struo versions annotate approximately the same number of genes. D) The percent of CPU hours saved via the Struo2 database updating algorithm versus de novo database generation. The original database was constructed from 1000 genomes. For B) and D), the lines are linear regressions, and the grey regions represent $95 \%$ confidence intervals. 
A

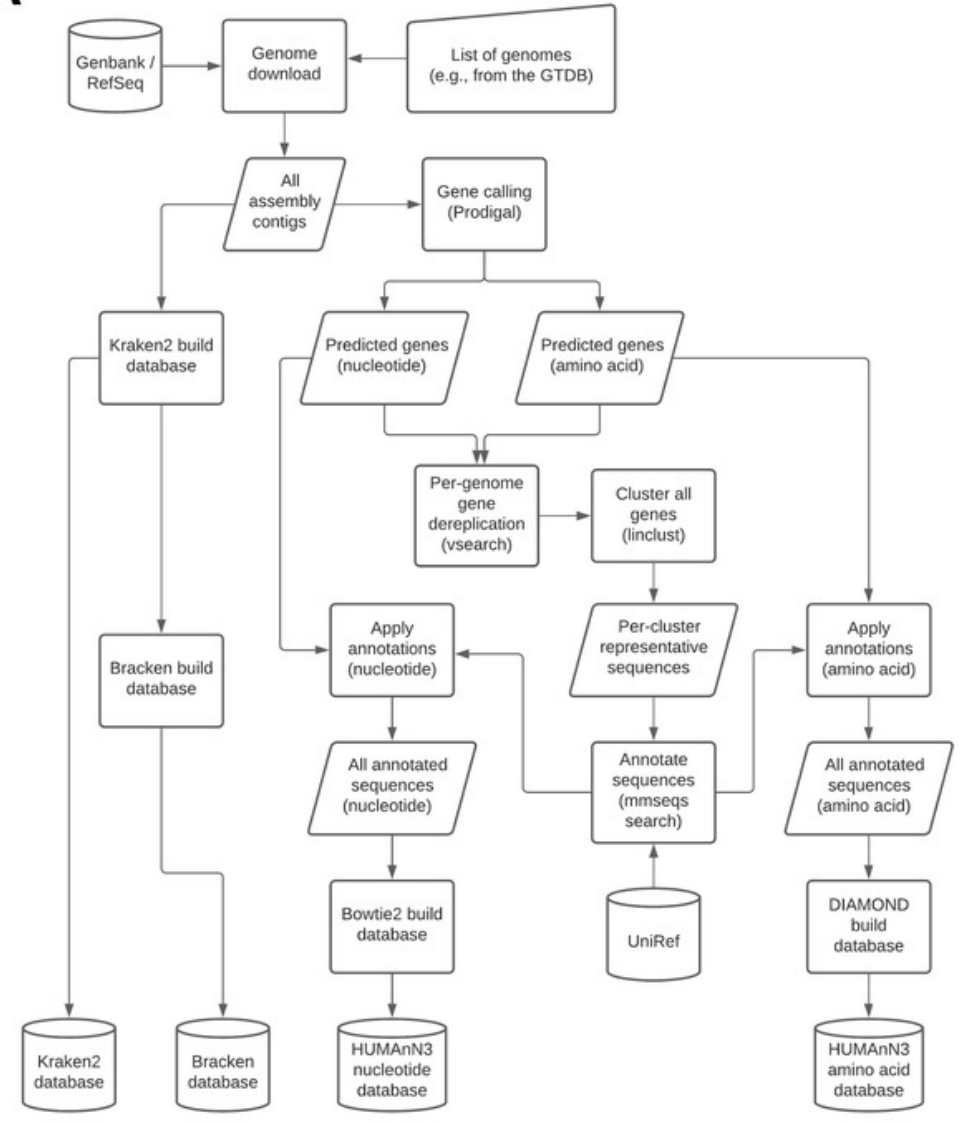

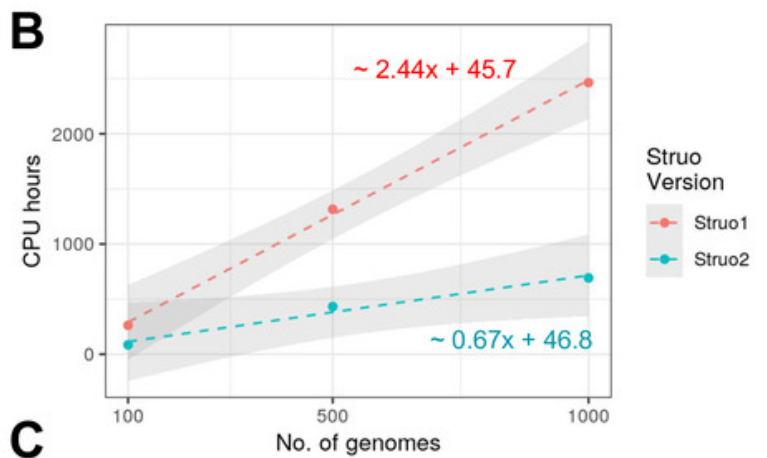
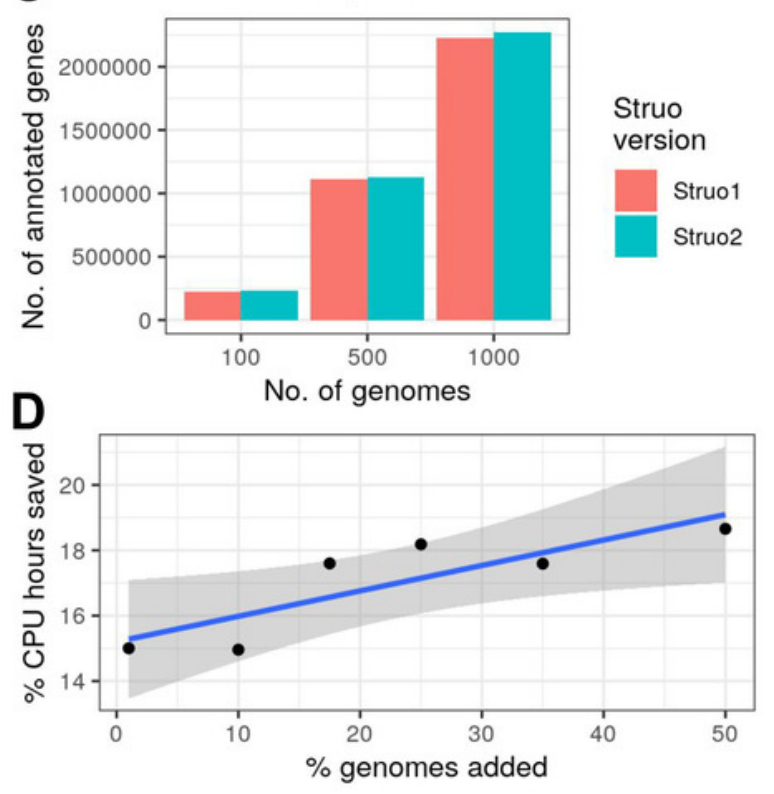


\section{Figure 3}

Struo2-generated gene database quality is substantially affected by reference genome assembly quality

Two reference genome datasets of 100 randomly selected genomes each ("n100_r1" and "n100_r2") were used for simulating misassemblies among all genomes in order to assess how genome assembly quality affects Struo2-generated database quality. "Ground truth" is the unaltered reference genomes, while the "bN-rN-cN" labels denote synthetic datasets with specific numbers of added misassemblies per genome (see Methods). A) CheckM-estimated assembly quality for each genome. B) The percent of genes annotated in the Struo2 database versus the ground truth. C) The percent of genes annotated correctly (i.e., correct UniRef90 ID) versus the ground truth. D) Change in Bray-Curtis distances between the ground truth and synthetic datasets (measured via Mantel tests), with beta diversity calculated from Bracken taxonomic assignments. The CAMI2 "HMP-gut" dataset of 10 metagenomes was used for benchmarking. 
A

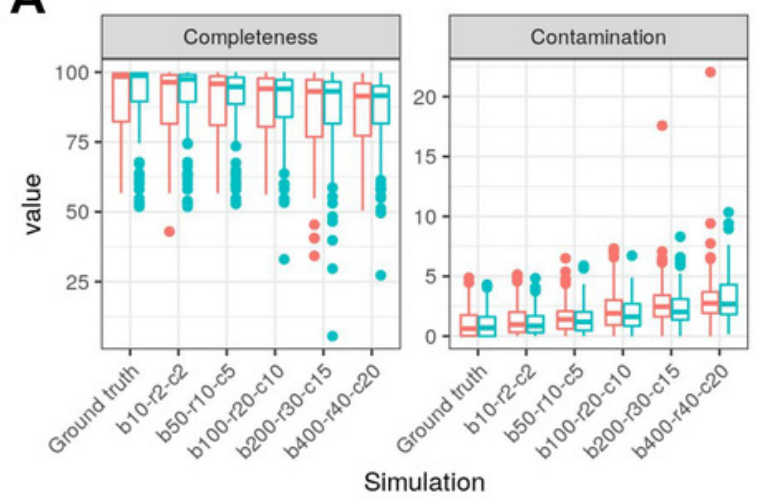

C

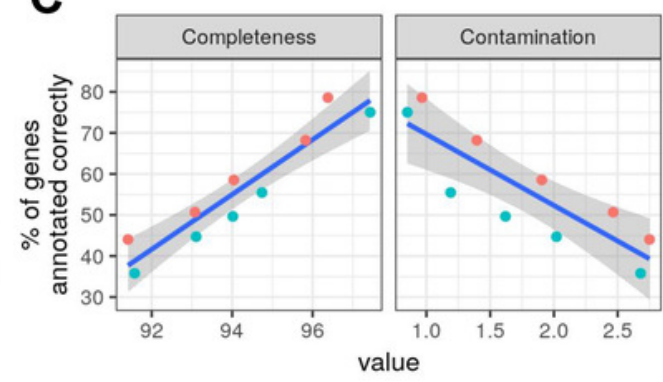

Reference genome dataset

- n100_r1

- n100_r2
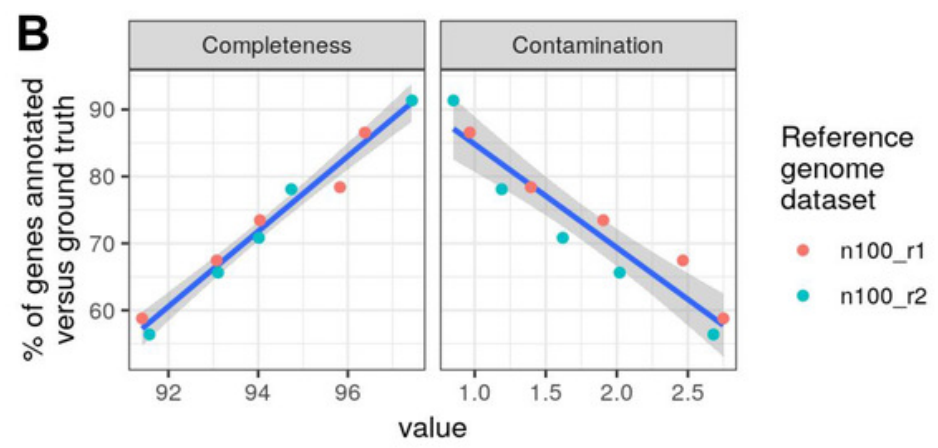

D

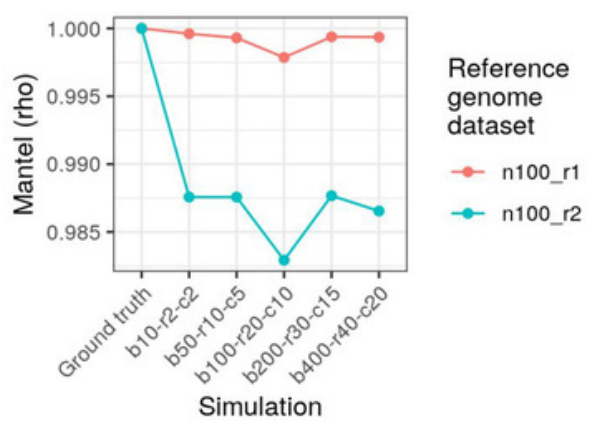

